\title{
ARTICLE Dorsomedial prefrontal cortex neurons encode nicotine-cue associations
}

Roeland F. Struik $\mathbb{D}^{1,2}$, Nathan J. Marchant ${ }^{1}$, Roel de Haan ${ }^{3}$, Huub Terra ${ }^{3}$, Yvar van Mourik ${ }^{1}$, Dustin Schetters ${ }^{1}$, Madison R. Carr ${ }^{1}$, Marcel van der Roest ${ }^{1}$, Tim S. Heistek ${ }^{3}$ and Taco J. De Vries ${ }^{1,2}$

The role of medial prefrontal cortex (MPFC) in regulating nicotine taking and seeking remains largely unexplored. In this study we took advantage of the high time-resolution of optogenetic intervention by decreasing (Arch3.0) or increasing (ChR2) the activity of neurons in the dorsal and ventral mPFC during 5-s nicotine cue presentations in order to evaluate their contribution to cued nicotine seeking and taking. Wistar rats were trained to self-administer intravenous nicotine in $1 \mathrm{~h}$ self-administration sessions twice a day for a minimum of 10 days. Subsequently, dmPFC or vmPFC neuronal activity was modulated during or following presentation of the 5-s nicotine cue, both under extinction and self-administration conditions. We also used in vivo electrophysiology to record the activity of dmPFC neurons during nicotine self-administration and extinction tests. We show that optogenetic inhibition of dmPFC neurons during, but not following, response-contingent presentations of the nicotine cue increased nicotine seeking. We found no effect on nicotine self-administration or on food seeking in an extinction test. We also show that this effect is specific to dmPFC, because optogenetic inhibition of vmPFC had no effect on nicotine seeking and taking. In vivo recordings revealed that dmPFC network neuronal activity was modulated more strongly following nicotine cue presentation in extinction, compared to following nicotine self-administration. Our results strongly suggest that a population of neurons within the dmPFC is involved in encoding the incentive value of nicotine-associated cues.

Neuropsychopharmacology (2019) 44:2011-2021; https://doi.org/10.1038/s41386-019-0449-x

\section{INTRODUCTION}

Nicotine addiction is one of the leading causes of preventable disease [1]. Current treatment strategies involve replacement therapy (e.g. nicotine gum) that, while effective at reducing smoking, do not address the underlying addiction to nicotine. In individuals that are abstinent from nicotine use, cues in the environment that are associated with tobacco use can induce craving and relapse [2]. This occurs through associative learning mechanisms, where a neutral stimulus repeatedly paired with a drug of abuse becomes a predictor for the drug reward, can elicit drug-seeking behavior, and can gain motivational properties itself [3-5].

Clinical imaging studies have identified the prefrontal cortex as a brain region that shows activity associated with cue-induced craving in smokers $[2,6]$. The role of a drug-paired cue in promoting relapse is examined in rodents using the cue-induced reinstatement model of relapse to drug seeking [3, 5]. These studies have previously identified a critical role for the medial prefrontal cortex (mPFC) in reinstatement of many drugs of abuse [3]. Studies on the role of the MPFC in nicotine seeking are scarce, however neural activity in dorsal mPFC is associated with the expression of cue-associated nicotine memories $[7,8]$. Recently we demonstrated a GABAergic mechanism in dorsal mPFC that controls cue-induced reinstatement of nicotine seeking [9].
The rodent mPFC is comprised of anatomically distinct dorsal and ventral regions [10]. While there is much evidence supporting a role for dorsal $\mathrm{mPFC}$ in promoting reinstatement of extinguished drug seeking (see above), the ventral mPFC has been implicated in mediating the extinction of drug and reward seeking [11, 12]. However, there is also substantial evidence showing that ventral mPFC manipulations can decrease drug seeking [13]. As such there is debate about the role of these brain regions in the control of drug seeking by drug-associated cues $[14,15]$. With respect to nicotine seeking, we have found that pharmacological inactivation of either ventral or dorsal mPFC decreased cue-induced reinstatement of extinguished nicotine seeking [9].

Long-lasting drug-dependent changes are both timedependent and brain-region specific and can contribute to reinstatement [16-19]. However, quick-onset and short-lasting changes have also been shown to be critical for reinstatement $[9,20,21]$. Furthermore, interventions that span the entire reinstatement test, can have opposite effect of interventions that cause transient changes $[9,22,23]$. To address this limitation we used the expression of microbial opsins Channelrhodopsin-2 (ChR2; [24]) or Archaerhodopsin 3.0 (Arch3.0; [25]) to manipulate the activity of CaMKIla expressing mPFC neurons specifically during the nicotine cue presentations in tests for nicotine seeking. In this study, we have used optogenetics to manipulate the

\footnotetext{
${ }^{1}$ Department of Anatomy and Neurosciences, Amsterdam Neuroscience, VU University Medical Centre, Amsterdam, the Netherlands; ${ }^{2}$ Center for Neurogenomics and Cognitive Research, Department of Molecular and Cellular Neurobiology, Amsterdam Neuroscience, VU University, Amsterdam, the Netherlands and ${ }^{3}$ Center for Neurogenomics and Cognitive Research, Department of Integrative Neurophysiology, Amsterdam Neuroscience, VU University, Amsterdam, the Netherlands

Correspondence: Taco Vries (tj.devries@vumc.nl)

These authors contributed equally: Roeland F. Struik, Nathan J. Marchant
}

Received: 6 March 2019 Revised: 29 May 2019 Accepted: 17 June 2019

Published online: 26 June 2019 
function of dmPFC and vmPFC neurons during nicotine cue presentation to identify their involvement in encoding nicotineassociated cues.

\section{MATERIALS AND METHODS}

Experimental animals

We obtained 134 male Wistar Unilever rats (weighing 225-300 g upon arrival) from either Harlan CPB (Horst, The Netherlands) or Charles River Laboratories B.V. (Leiden, The Netherlands). All behavioral procedures were performed during the dark-phase of the diurnal cycle, food and water was available ad libitum throughout. All animal biotechnical and behavioral procedures were approved by the Animal Experiments Committee of the Vrije Universiteit, Amsterdam, The Netherlands.

\section{Surgery}

Intravenous catheter and intracranial surgery. Prior to surgery, Intravenous (i.v.) catheters were constructed as documented in De Vries et al., [26] and chronically implantable optic fibers were made as reported by Sparta et al. [27] (see also Supplementary Information). Our i.v. surgery was performed as described previously [26] (see also Supplementary Information). We bilateraIly injected the virus solution $(0.5 \mu \mathrm{l})$ into dmPFC (Anterio-Posterior (AP): $+2.76 \mathrm{~mm}$; Medio-Lateral (ML): $\pm 1.35 \mathrm{~mm}$; Dorso-Ventral (DV): $-3.45 \mathrm{~mm}$, at a $10^{\circ}$ angle) or vmPFC (AP: $+2.80 \mathrm{~mm}$; ML: \pm $1.50 \mathrm{~mm}$; DV: $5.40 \mathrm{~mm}$, at a $10^{\circ}$ angle) at an infusion rate of $0.1 \mathrm{\mu l} / \mathrm{min}$. The viral constructs were: $\mathrm{rAAV} 5 /$ CaMKIla-eArch3.0eYFP $\left(2.5 \times 10^{12}\right.$ viral genomes/ml), rAAV5/CaMKlla-eYFP (5.2 or $6.3 \times 10^{12}$ viral genomes/ml) or rAAV5/CaMKIla-hChR2(H134R)eYFP-WPRE $\left(8.5 \times 10^{12}\right.$ viral genomes $\left./ \mathrm{ml}\right)$ provided by the Gene Therapy Center Vector Core (University of North Carolina, Chapel Hill, NC, USA).

For the dmPFC experiments, we implanted the tip of the fibers $0.4 \mathrm{~mm}$ above the virus injection site (AP: $+2.76 \mathrm{~mm}$; ML: \pm $1.28 \mathrm{~mm}\left(10^{\circ}\right.$ angle); DV: $\left.-3.05 \mathrm{~mm}\right)$. For the last 18 surgeries of the dmPFC ChR2 and eYFP infusions, the AP coordinate for both the virus infusion and fiber implantation was changed to $A P:+2.6$. For the vmPFC experiment, we implanted the optic fibers approximately $0.5 \mathrm{~mm}$ above the virus injection site (AP: $+2.80 \mathrm{~mm}, \mathrm{ML}: \pm 1.50 \mathrm{~mm}\left(10^{\circ}\right.$ ngle), DV: $\left.-4.90 \mathrm{~mm}\right)$.

In vivo electrophysiology surgery. We first implanted the i.v. silicon catheters as above, with the distal end of the catheter attached to a connector pedestal (Plastics One Inc., Minneapolis, MN, USA) anchored between the shoulder blades. We then trained the rats for nicotine self-administration prior to the microdrive surgery. During the microdrive surgery, an in our lab improved version of the Harlan 4 Drive (Neuralynx, Bozeman, MT, USA) was placed above the mPFC (from Bregma: AP: $+2.6 \mathrm{~mm}$; ML: $-0.7 \mathrm{~mm}$ ) and the tetrodes were lowered to $3.0 \mathrm{~mm}$ below cortical surface (Supplementary Information).

Behavioral procedures

Nicotine self-administration. Nicotine self-administration was performed as described in De Vries et al., [26] (for details see Supplementary Information), except as follows. We trained rats to self-administer nicotine for a minimum of 10 days, two $1 \mathrm{~h}$ sessions per day, five days a week, with a minimum interval of $2 \mathrm{~h}$ between sessions (sessions 1: 09.00-14:30, sessions 2: 13:00-19:00). Upon nose-poking in the active hole (the position of the active and inactive hole was counterbalanced between rats), nicotine (nicotine hydrogen tartrate salt, SigmaAldrich, St. Louis, MO, USA) in saline $(\mathrm{pH}=7.4)$ was infused over approximately $2 \mathrm{~s}$ (infusion time adjusted for weight) at $40 \mu \mathrm{g} / \mathrm{kg} /$ infusion. The nicotine infusion began after a $0.5-\mathrm{s}$ delay, upon which a tone is played and the nose-poke hole is illuminated for $5 \mathrm{~s}$ (Nicotine cue). There was a 15-s time-out period, during which responses in either hole are recorded but are without consequence.

For details on laser set-up and optical tethering, see Supplementary Information.

\section{Stimulation parameters}

Light transmission of the optical tether was measured before and after each stimulation session using a fiber optic power meter (Thorlabs Inc., Newton, NJ, USA). The Arch3.0 stimulation parameters consisted of $532 \mathrm{~nm}$ wavelength continual light with an approximate intensity of $\sim 10 \mathrm{~mW}$ (dmPFC) or $\sim 5 \mathrm{~mW}$ (vmPFC) and as measured using an unimplanted fiber of $70 \%$ light transmission. The ChR2 stimulation parameters consisted of pulsed $473 \mathrm{~nm}$ wavelength light with an intensity of $4-6 \mathrm{~mW}$ as measured using an unimplanted fiber of $70 \%$ light transmission $(20 \mathrm{~Hz}, 10 \mathrm{~ms}$ pulse length, $40 \mathrm{~ms}$ interpulse interval, 120 pulses over $6 \mathrm{~s}$ ). ChR2 stimulation parameters were chosen based on the importance of beta oscillations in cue utilization and the success of $20 \mathrm{~Hz}$ pulses to induce Fos expression in mPFC pyramidal neurons $[18,28]$.

\section{Immunohistochemistry}

After completion of behavioral testing, rats were transcardially perfused with $4 \%$ paraformaldehyde (PFA; Sigma-Aldrich, St. Louis, MO, USA).

Fixed brains were sliced at $40 \mu \mathrm{m}$ by a sliding microtome (Rankin biomedical, AO 860) and stored in 1x PBS with $0.02 \%$ sodium azide. Slices were fluorescently stained for eYFP, as a reporter of viral expression and the neuronal marker NeuN, as previously described [29] (for details see Supplementary Information).

In vitro electrophysiology. We employed patch-clamping techniques to determine Arch3.0 functionality and the physiological effects of using Arch3.0 to inhibit cell firing (for details see Supplementary Information and Fig. S1). Light-induced hyperpolarization was characterized in Arch3.0 expressing mPFC neurons under current clamp conditions following laser light exposure at a light intensity range $(0,1.7,3,7,12 \mathrm{~mW})$ and a pulse duration range $(100,500,6000 \mathrm{~ms})$.

\section{Behavioral experiments}

Exp. 1: Effect of optogenetic inhibition of dmPFC on nicotine seeking $(n=53)$. We injected rats either with AAV encoding Arch3.0 or eYFP in the dmPFC and then trained them to self-administer nicotine. On the last day of self-administration, rats were trained to self-administer nicotine during the morning session and had an extinction session in the afternoon with one of the following two optical intervention protocols: [1] on cue: $6 \mathrm{~s}$ of responsecontingent inhibition, encompassing the 5 -s cue and $0.5 \mathrm{~s}$ before and after this cue; or [2] off cue: $6 \mathrm{~s}$ of optical inhibition occurring $60 \mathrm{~s}$ after a cue-reinforced nose-poke (see Fig. 1c). The 60-s period was chosen so that cue exposure did not consistently coincide with optical inhibition. The following morning, the rats had an extinction test without optical intervention. Following nicotineextinction testing, the effect of on cue inhibition on nicotine selfadministration and food pellet extinction was tested (for detailed description see Supplementary Information). For the combined nicotine self-administration and optical intervention in Exp. 1 and Exp. 3, tangling of the nicotine tubing with the optical tether was prevented by leading the two optic fibers and the nicotine tubing through a single metal spring attached to a commutator with sufficient length of tubing outside of the rats' reach to allow for turning with minimal hindrance to the rats' movement. Nicotine self-administration response rates in rats expressing an opsin were compared to response rates in rats expressing eYFP under the same experimental conditions, including possible hindrances to movement, if any. 
A

\section{Behavioral procedure}

\begin{tabular}{|c|c|c|}
\hline $\begin{array}{l}\text { Nicotine } \\
\text { self-admin }\end{array}$ & Test 1: & Test 2: \\
\hline $2 \times 1$ h per day & Morning: & Morning: \\
\hline $\begin{array}{c}40 \text { ug/kg/inf } \\
\text { FR-1 }\end{array}$ & $\begin{array}{c}1 \mathrm{~h} \text { nic self-admin } \\
\text { Afternoon: } \\
1 \mathrm{~h} \text { extinction test } \\
\text { With laser }\end{array}$ & $\begin{array}{l}1 \mathrm{~h} \text { extinction test } \\
\text { Without laser }\end{array}$ \\
\hline
\end{tabular}

B



C

Optogenetic test parameters



Fig. 1 Optogenetic inhibition of dmPFC neurons during cue exposure increases nicotine seeking. a Outline of experimental procedure. b Mean \pm sem number of nose-pokes during the nicotine self-administration sessions prior to the extinction tests (Arch on cue: $n=8$; eYFP on cue: $n=9$; Arch off cue: $n=10$; eYFP off cue: $n=8$ ). c Schematic of the two different test conditions of optogenetic inhibition of dmPFC neurons. d Mean \pm sem number of nose-pokes during test during the on cue stimulation of Arch3.0 test (left) and on the extinction test the following day (right) (eYFP: $n=9$; Arch3.0: $n=8 ;^{* *}=p<0.01 ;{ }^{* * *}=p<0.005$ ). e Mean \pm sem number of nose-pokes during test during the off cue stimulation of Arch3.0 test (left) and on the extinction test the following day (right) (eYFP: $n=8 ;$ Arch3.0: $n=10$ )

Exp. 2: Effect of optogenetic stimulation of dmPFC on nicotine seeking $(n=41)$. We injected rats either with AAV encoding ChR2 or eYFP into the dmPFC, and then trained them to self-administer nicotine. Test day is similar to experiment 1 , where the on cue test condition is $6-\mathrm{s} 20 \mathrm{~Hz}$ stimulation immediately after a cue-reinforced nose-poke and the off cue stimulation is 6-s $20 \mathrm{~Hz}$ stimulation $60 \mathrm{~s}$ after a cue-reinforced nose-poke. Consecutively, rats were tested for the effects of on cue stimulation on nicotine self-administration and food pellet extinction in separate trials (for detailed description see Supplementary Information), 
Exp. 3: Effect of optogenetic inhibition of vmPFC on nicotine seeking $(n=24)$. We injected rats with either AAV encoding Arch3.0 or eYFP in vmPFC, and then trained them to self-administer nicotine. Rather than test the two optical intervention protocols (on cue, off cue) between-subjects, we used a repeated testing, withinsubjects design where the test order was counterbalanced between groups. After 12 days of nicotine self-administration, on the afternoon of the test day, one group received on cue inhibition of VmPFC and the other group received off cue inhibition of vmPFC. After eight retraining nicotine selfadministration sessions, the rats were tested again and the test day treatment was reversed. Each test day was followed by an extinction memory test on the following morning.

Exp. 4: Effect of nicotine seeking in extinction on dmPFC neuron activity $(n=3)$. In this experiment we used in vivo electrophysiology to record neuronal activity in dmPFC during nicotine selfadministration and extinction tests. We recorded dmPFC neuronal activity with neurophysiological recordings during nicotine and extinction sessions. After each session pair, tetrodes were lowered $80-160 \mu \mathrm{m}$. We re-trained the rats until a stable level of nicotine intake was achieved and they were then recorded again in a nicotine and extinction session. For further information on the neurophysiological recordings see Supplementary Information.

\section{Statistics}

All statistical analysis on behavior was performed using IBM Statistical Package for the Social Sciences (SPSS) version 22 (for detailed description see Supplementary Information). In short, for each behavioral test, we first performed a two-factor repeated measures ANOVA over the self-administration period with the within-subjects factors Time and Nose-poke and the betweensubjects factor Group (Arch3.0 or ChR2, eYFP) followed up by Fisher's least significant difference post-hoc test to determine if there are differences between groups during self-administration. We then performed a two-factor repeated measures ANOVA over the first and second test day with the within-subject factors Test Day and Nose-poke and the between-subjects factor Group (Arch3.0 or ChR2, eYFP) followed up Fisher's least significant difference post-hoc test and one-way ANOVA.

For the in vivo electrophysiology experiments, all data analysis was performed off-line on recording sessions with four or more active nose-pokes. Single-units were isolated by cluster-cutting analysis using Offline Sorter V4 (Plexon Inc, Dallas, TX, USA). Further analysis was done using NeuroExplorer 4 (Nex Technologies, Colorado Springs, CO, USA) and custom scripts written in MATLAB. Firing rates were calculated per trial based on the median amount of spikes $0-3 \mathrm{sec}$ after nose-poke, the median was taken over the trials per neuron. $\Delta$ Firing rates were calculated by subtracting the session mean firing rate and Z-scores were made based on firing rate and standard deviation of the whole session. Medians and variances between groups were compared using a non-parametric Mann-Whitney $U$ test and Levene's (absolute deviations from group means) test, respectively.

\section{RESULTS}

Exp. 1: Optogenetic inhibition of dmPFC neurons during nicotine cue presentations increases nicotine seeking

Effect of 6-s optogenetic inhibition of dmPFC neurons during nicotine-cue presentations in an extinction test; (eYFP: $n=9$, Arch3.0: $n=8$ ). To determine the role of dmPFC in cued nicotine seeking under extinction conditions, we inhibited dmPFC neurons during the cue exposure phase of an extinction session and tested for extinction memory sixteen hours later (Fig. 1a). Figure S5A shows example expression of Arch3.0 in dmPFC, and fiber placements above dmPFC. Figure $1 \mathrm{~b}$ shows the nicotine self-administration data. Some rats received more optic fiber tether habituation sessions than others, as such the total number of nicotine selfadministration sessions was not identical for all rats. However, there was no difference between the groups in the total number of sessions: group eYFP, $39.1 \pm 3.1$; group Arch3.0, 39.3 \pm 4.4 (mean sessions \pm sem).

Figure $1 \mathrm{~d}$ shows total nose-pokes during the two test days for the rats that received optogenetic inhibition of dmPFC during nicotine cue presentation. We found a significant effect of Test Day $(F(1,30)=9.3, p<0.01)$, and significant interaction between Test Day and Group $(F(1,30)=9.9, p<0.005)$ and Test Day and Nose-poke $(F(1,30)=9.141, p<0.01)$. One-way ANOVA comparing active responding on Test Day 1 proved a significant difference between Groups $(F(1,15)=11.633, p<0.005)$. This reflects an increase in nicotine seeking observed during the cued extinction test in the Arch3.0 group compared to the eYFP control group. We found no interaction between Test Day, Nose-poke, and Group $(F(1,30)=2.383, p>0.05)$. These data show that optogenetic inhibition of CaMKIla neurons in dmPFC during nicotine cue presentations increases nicotine seeking. Figure S2 shows the accumulative plot of within-session responding during the optogenetic inhibition test. It clearly shows that while control rats plateau after $\sim 15 \mathrm{~min}$, rats that received inhibition of dmPFC neurons during cue presentations keep responding throughout the session.

Effect of 6-s optogenetic inhibition of dmPFC neurons $60 \mathrm{~s}$ after nicotine-cue presentations in an extinction test; (eYFP: $n=8$, Arch3.0: $n=10$ ). Figure $1 \mathrm{~b}$ shows nicotine self-administration, and Fig. 1e shows total nose-pokes during the two test days for the rats that received optogenetic inhibition of dmPFC $60 \mathrm{sec}$ after nicotine cue presentation. The rats were tested in an identical manner to the previous group (Fig. 1c). Average number of nicotine selfadministration sessions was not identical for all rats, but did not differ between the groups (eYFP: $29.5 \pm 3.0$, Arch3.0: $30.7 \pm 2.0$ ). Statistical analysis revealed no significant effect of Test Day, Nosepoke, or Group, and there were no significant interactions ( $\mathrm{Fs}<1$; ps $>0.05$ ). These data show that optogenetic inhibition of CaMKIla neurons in dmPFC outside the nicotine cue presentation window has no effect on nicotine seeking.

Optogenetic inhibition during cue presentations did not affect responding in rats that were self-administering nicotine (Fig. S3B), or in rats responding for a food cue during an extinction test (Fig. S3D).

Exp. 2: Optogenetic stimulation of dmPFC neurons during nicotine cue presentations has no effect on nicotine seeking

Effect of 6-s optogenetic stimulation of dmPFC neurons during nicotine-cue presentations in an extinction test; (eYFP: $n=7$, ChR2: $n=6)$. To determine whether activation of dmPFC neurons can suppress nicotine seeking we used ChR2 to stimulate the dmPFC at a $20 \mathrm{~Hz}$ frequency during nicotine cue presentation (Fig. 2a). Figure S5B shows example expression of ChR2 in dmPFC, and fiber placements above dmPFC. Figure $2 b$ shows the nicotine selfadministration performance. The average number of nicotine selfadministration sessions were: group eYFP, $40.0 \pm 1.3$; group ChR2, $41.3 \pm 1.2$ (mean sessions \pm sem).

Figure $2 \mathrm{~d}$ shows total nose-pokes during the two test days for the rats that received optogenetic stimulation of dmPFC during nicotine cue presentation. We analyzed the response rates using the same statistical approach as for Exp. 1. We found no significant effect of Test Day $(F(1,22)=0.530, p>0.05)$ and no significant interaction between Test Day and Group $(F(1,22)=$ $1.562, p>0.05)$. Furthermore we found no significant interactions between Test Day and Nose-poke $(F(1,22)=1.096, p>0.05)$ and Test Day, Group, and Nose-poke $(F(1,22)=3.035, p>0.05)$. These data show that optogenetic stimulation of CaMKIla neurons in dmPFC during presentation of the nicotine cue has no effect on nicotine seeking. 
A

\section{Behavioral procedure}

\begin{tabular}{|c|c|c|}
\hline $\begin{array}{l}\text { Nicotine } \\
\text { self-admin }\end{array}$ & Test 1: & Test 2: \\
\hline $2 \times 1$ h per day & Morning: & Morning: \\
\hline $\begin{array}{c}40 \mathrm{ug} / \mathrm{kg} / \mathrm{inf} \\
\text { FR-1 }\end{array}$ & $\begin{array}{l}1 \mathrm{~h} \text { nic self-admin } \\
1 \mathrm{~h} \text { Afternoon: } \\
\text { extinction test } \\
\text { With laser }\end{array}$ & $\begin{array}{l}1 \mathrm{~h} \text { extinction test } \\
\text { Without laser }\end{array}$ \\
\hline
\end{tabular}

B

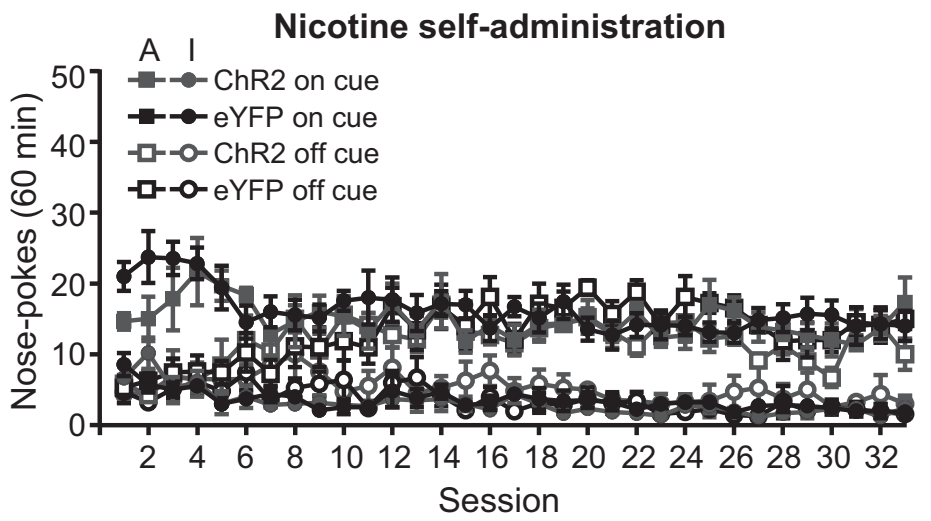

C

Optogenetic test parameters

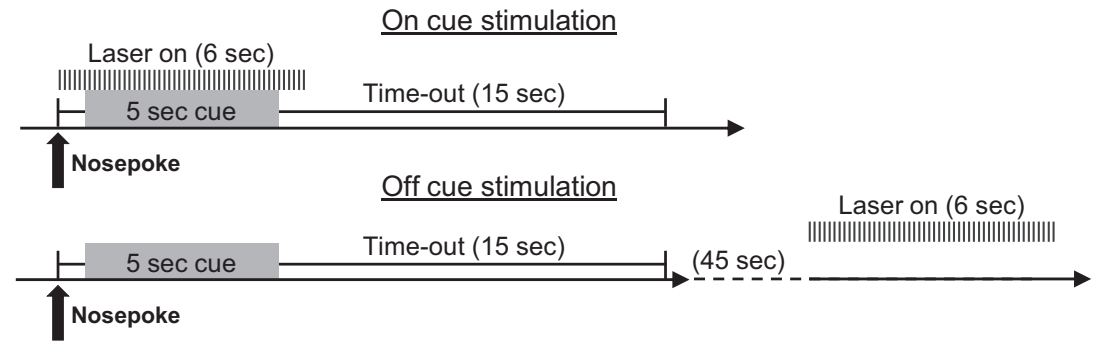

Dicotine extinction test dmPFC stimulation on cue

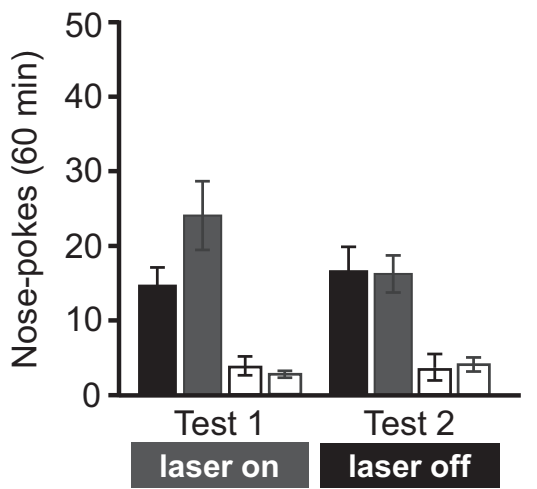

E Nicotine extinction test dmPFC stimulation off cue

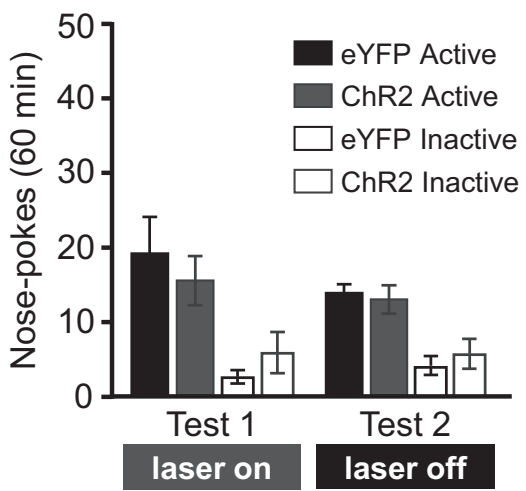

Fig. 2 Optogenetic excitation of dmPFC neurons during cue exposure has no effect on nicotine seeking. a Outline of experimental procedure. b Mean \pm sem number of nose-pokes during the nicotine self-administration sessions prior to the extinction tests (ChR2 on cue: $n=6$; eYFP on cue: $n=7$; ChR2 off cue: $n=7$; eYFP off cue: $n=7$ ). c Schematic of the two different test conditions of optogenetic excitation of dmPFC neurons. d Mean \pm sem number of nose-pokes during test during the on cue stimulation of ChR2 test (left) and on the extinction test the following day (right) (eYFP: $n=7$; ChR2: $n=6$ ). e Mean \pm sem number of nose-pokes during test during the off cue stimulation of ChR2 test (left) and on the extinction test the following day (right) (eYFP: $n=7$; ChR2: $n=7$ )

Effect of 6-s optogenetic stimulation of dmPFC neurons $60 \mathrm{~s}$ after nicotine-cue presentations in an extinction test; (eYFP: $n=7$, ChR2: $n=7)$. Figure $2 \mathrm{~b}$ shows nicotine self-administration, and Fig. $2 \mathrm{e}$ shows total nose-pokes during the two test days for the rats that received optogenetic stimulation of dmPFC $60 \mathrm{sec}$ after nicotine cue presentation. The rats were tested in an identical manner to the previous group (Fig. 2c). Statistical analysis revealed no significant effect of Test Day $(F(1,24)=1.448, p>0.05)$, Test Day by Nose-poke interaction $(F(1,24)=2.574, p>0.05)$, or Test Day by Group interaction $(F(1,24)=0.063, p>0.05)$, and no Test Day by 


\begin{tabular}{|c|c|c|c|c|c|}
\hline $\mathbf{A}$ & & Behavioral pr & ocedure & & \\
\hline $\begin{array}{l}\text { Nicotine } \\
\text { self-admin }\end{array}$ & Test 1: & Test 2: & $\begin{array}{l}\text { Nicotine } \\
\text { self-admin }\end{array}$ & Test 1: & Test 2: \\
\hline $\begin{array}{c}2 \times 1 \text { h per day } \\
40 \mathrm{ug} / \mathrm{kg} / \mathrm{inf} \\
\text { FR-1 }\end{array}$ & $\begin{array}{l}1 \mathrm{Morning:} \\
1 \mathrm{~h} \text { nic self-admin } \\
1 \mathrm{Afternoon:} \\
1 \mathrm{~h} \text { extinction test } \\
\text { With laser }\end{array}$ & $\begin{array}{l}\text { Morning: } \\
1 \mathrm{~h} \text { extinction test } \\
\text { Without laser } \\
1 \mathrm{Afternoon:} \\
\text { nic self-admin }\end{array}$ & $\begin{array}{c}2 \times 1 \text { h per day } \\
40 \mathrm{ug} / \mathrm{kg} / \mathrm{inf} \\
\mathrm{FR}-1\end{array}$ & $\begin{array}{l}1 \mathrm{~h} \text { Morning: } \\
1 \mathrm{~A} \text { Afternoon: } \\
1 \mathrm{~h} \text { extinction test } \\
\text { With laser }\end{array}$ & $\begin{array}{l}\text { Morning: } \\
1 \mathrm{~h} \text { extinction test } \\
\text { Without laser }\end{array}$ \\
\hline
\end{tabular}

B

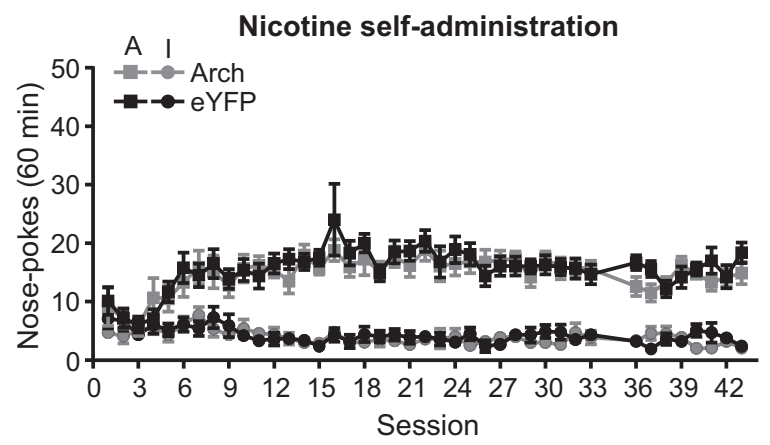

C

Optogenetic test parameters
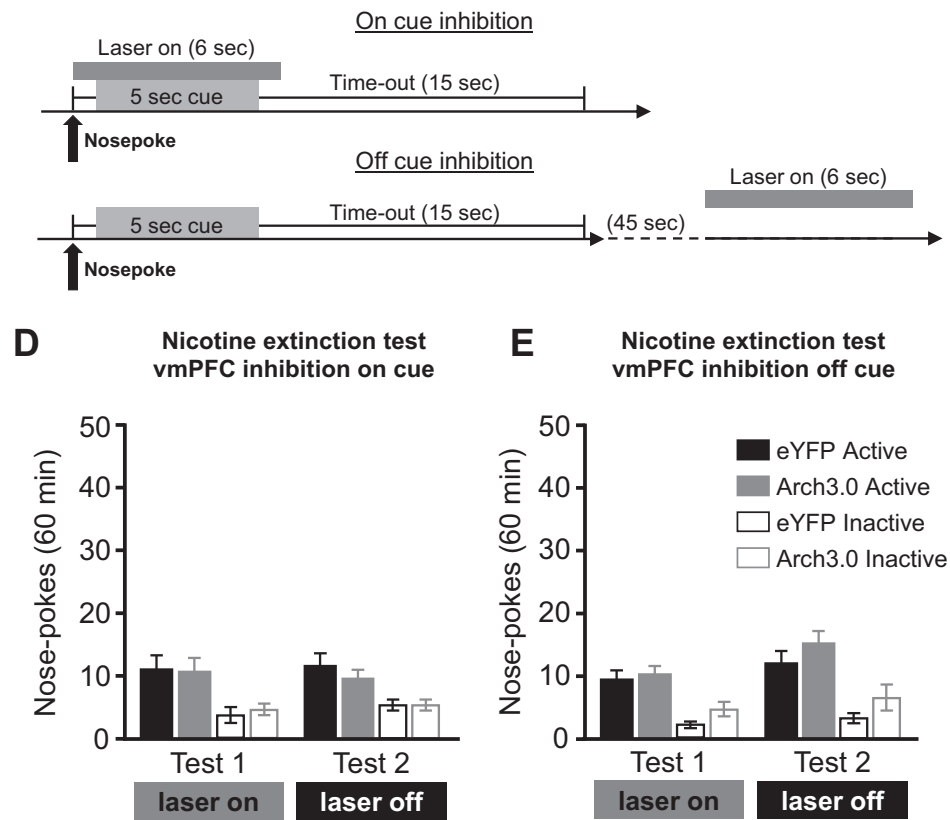

Fig. 3 Optogenetic inhibition of vmPFC neurons during cue exposure has no effect on nicotine seeking. a Outline of experimental procedure. b Mean \pm sem number of nose-pokes during the nicotine self-administration sessions prior to the extinction tests (eYFP: $n=10 ;$ Arch: $n=9$ ). c Schematic of the two different test conditions of optogenetic inhibition of dmPFC neurons. d Mean \pm sem number of nose-pokes during the on cue stimulation of Arch3.0 test (left) and on the extinction test the following day (right) (eYFP: $n=10 ;$ Arch3.0: $n=9$ ). e Mean \pm sem number of nose-pokes during the off cue stimulation of Arch3.0 test (left) and on the extinction test the following day (right) (eYFP: $n=10$; Arch3.0: $n=9$ )

Nose-poke by Group interaction $(F(1,24)=0.566, p>0.05)$.

Optogenetic stimulation during cue presentations had no effect on responding in rats that were self-administering nicotine (Fig. S4B), or in rats responding for a food-associated cue during an extinction test (Fig. S4D).

Exp. 3: Optogenetic inhibition of vmPFC neurons during nicotine cue presentations has no effect on nicotine seeking

Effect of 6-s optogenetic inhibition of vmPFC neurons during nicotine-cue presentations in an extinction test; (eYFP: $n=10$, Arch3.0: $n=9$ ). To determine the role of vmPFC in nicotine seeking, we inhibited vmPFC neurons during the nicotine cue exposure phase of an extinction session and tested for extinction memory $16 \mathrm{~h}$ later (Fig. 3a). Figure S5C shows example expression of Arch3.0 in vmPFC, and fiber placements above vmPFC. Figure $3 \mathrm{~b}$ shows the nicotine self-administration data. In this experiment, we tested rats in both test conditions (on cue, off cue), and the order of test was counterbalanced. The first test occurred after 33 sessions, and all rats were then trained for another eight sessions and tested again for a nicotine-extinction and extinction memory test (test 2).

Figure $3 d$ shows nicotine seeking during vmPFC inhibition on cue. We found no effect of Test Day $(F(1,17)=0.065, p>0.05)$, and no interactions between Test Day and Group $(F(1,17)=$ $0.221, p>0.05)$ and Nose-poke $(F(1,17)=1.074, p>0.05)$. Finally, there was no interaction between Test Day, Nose-poke, and Group $(F(1,17)=0.007, p>0.05)$. These data show that optogenetic inhibition of CaMKIla neurons in vmPFC during 


\section{A}

Recording location

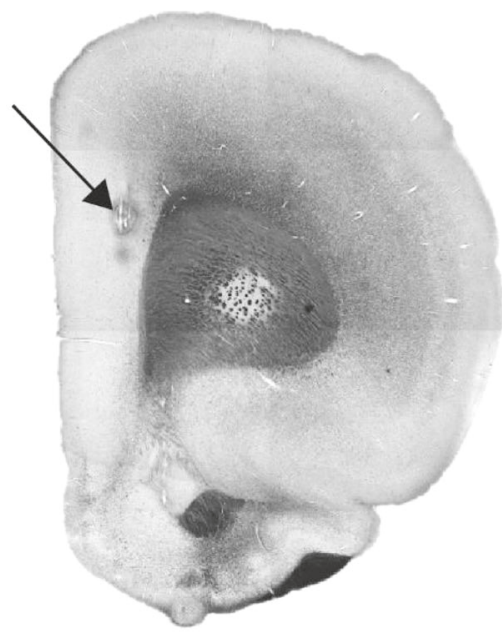

C

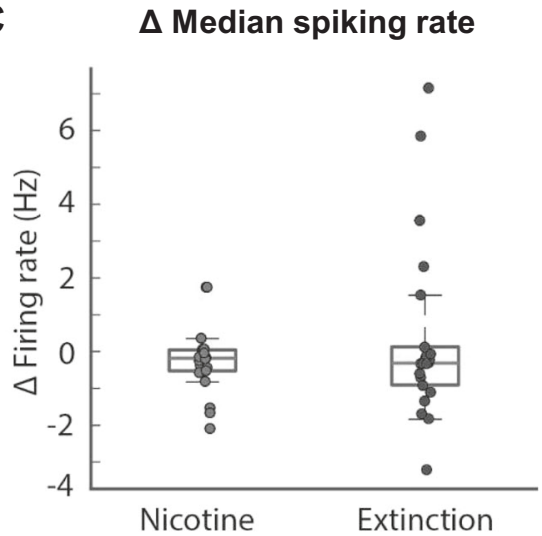

B

\section{Example traces}

Nicotine

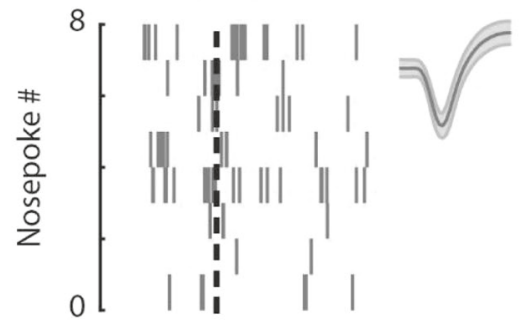

Extinction



D $\Delta$ z-score spiking rate

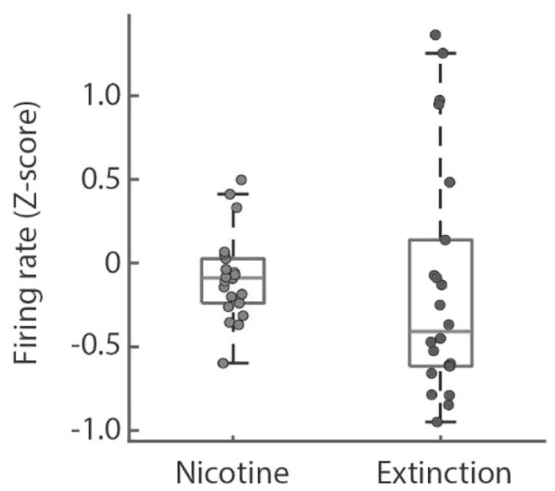

Fig. 4 Electrophysiological correlates of cued nicotine self-administration and extinction in dmPFC. a Example location of recording electrodes in the dmPFC. Arrow indicates recording location. b Raster plots and waveforms of two example units recorded during a nicotine self-administration (red) and extinction (blue) session aligned to nose-poke (dashed line). Waveforms are from a single lead and are represented as mean \pm std. $\mathbf{c}$, $\mathbf{d}$ Trial median firing rates per neuron $0-3 \mathrm{~s}$ after nose-poke in $\mathbf{c} \Delta \mathrm{Firing}$ rates $(\mathrm{Hz})$ from baseline and $\mathbf{d} \mathrm{Z}$-scored firing rates. Boxplot shows median \pm 1 st and 3rd quartile

presentation of the nicotine cue has no effect on nicotine seeking.

Effect of 6-s optogenetic inhibition of vmPFC neurons $60 \mathrm{~s}$ after nicotine-cue presentations in an extinction test; (eYFP: $n=10$, Arch3.0: $n=9$ ). Figure 3e shows nicotine seeking during vmPFC inhibition off cue. Using the same statistical approach, we found a main effect of Test Day $(F(1,17)=4.572, p<0.05)$, however we found no interaction between Test Day and Group $(F(1,17)=$ $0.444, p>0.05)$ and Nose-poke $(F(1,17)=2.137, p>0.05)$. Finally, we found no interaction between Test Day, Nose-poke, and Group $(F(1,17)=0.261, \quad p>0.05)$. Results indicate that there was enhanced nicotine seeking in the extinction test after the 'off cue' test session. However, this effect was not specific to the Arch3.0 group.

Exp. 4: Activity of putative pyramidal neurons in dmPFC during nicotine seeking

To determine a neurophysiological correlate of nicotine taking and seeking in dmPFC, we recorded spiking activity of dmPFC putative pyramidal neurons during nicotine self-administration and extinction. Well separated single-units were selected (peak-totrough-time $>0.7 \mathrm{~ms}$ [30], more than 300 spikes and less than $0.5 \%$ of inter-spike-intervals within $2 \mathrm{~ms}$ ). Tetrodes were kept at the same recording depth between pairs of nicotine selfadministration and nicotine-extinction session. In four session pairs from three rats behavioral and electrophysiological data was of sufficient quality. We recorded a total of 22 single-units per session type over an average of 5.25 and 8.25 trials/session for the nicotine and extinction group, respectively (Fig. 4b).

Figure $4 \mathrm{c}, \mathrm{d}$ show the $\Delta$ firing rates and Z-scored firing rates for the nicotine and extinction group 0 to $3 \mathrm{~s}$ after the nose-poke. Neither the population median of $\Delta$ firing rate (Nic; $n=22,-0.09$ $(-0.52,0.05)$, Ext; $n=22,(-0.91,0.13), p>0.05)$, nor of Z-scores (Nic; $n=22,-0.09(-0.24,0.026)$, Ext; $n=22,0.21(-0.35,0.09)$, $p>0.05$, Mann-Whitney $\mathrm{U}$ test, values represent median, (1st; 3rd quartile)) were significantly different between the nicotine and extinction groups. Nevertheless, Levene's tests showed that the variance of $\Delta$ firing rate $(F(1,42)=8.61, p=0.0054)$ and Z-scores $(F(1,42)=15.29, p=0.0003)$ responses was significantly different 
between groups, with a wider distribution in the extinction group compared to the nicotine group. These data indicate that during extinction of nicotine seeking, the activity of the dmPFC neuronal network is more strongly modulated than during nicotine selfadministration.

We were concerned that the unequal numbers of active nosepokes per group might confound these findings. To address this, we used a bootstrapping approach where we randomly down sampled trials per session to 4 trails, lower the cut-off for qualitatively acceptable sessions. We then recalculated a $p$-value for differences in population variance and repeated this randomization 1000 times. We report a median p-value over 1000 iterations of 0.01 for $\Delta$ firing rate and 0.06 for Z-scores (Fig. S6). These findings further support the interpretation that the dmPFC neuronal network as a whole is more responsive to extinction of nicotine seeking, compared to when nicotine is received during nicotine self-administration sessions.

\section{DISCUSSION}

Here we identify an important role for dmPFC neurons in encoding nicotine-associated cues. We showed that optogenetic inhibition of dmPFC neurons during nicotine cue presentation increased nicotine seeking under extinction conditions. This was specific to the cue presentation time window, because optogenetic inhibition after cue presentation had no effect. Furthermore, we showed that this manipulation had no effect on nicotine self-administration or on food seeking. In contrast, optogenetic stimulation of dmPFC neurons during cue presentation had no effect on nicotine seeking during extinction tests, during nicotine self-administration tests, and there was no effect on food seeking. Optogenetic inhibition of vmPFC had no effect on nicotine seeking during extinction tests. Finally, we used in vivo electrophysiology to show that dmPFC network neuronal activity is modulated more strongly during extinction than during nicotine self-administration. Our results demonstrate the existence of a neuronal population in the dmPFC involved in encoding discrete nicotine-associated cues. This study highlights the critical role of dmPFC neurons in encoding cues associated with nicotine, and that this activity is causally related to nicotine seeking.

\section{Methodological considerations}

In our approach we took advantage of the temporal precision of optogenetics and manipulated neuronal activity during nicotine cue presentation. This allows us to study a more direct link between neuronal activation during cue exposure and the associated behavioral response (nicotine seeking). In addition, it minimizes the risks of side-effects that are known to occur following prolonged light exposure. Evidence has come to light showing that sustained Arch photostimulation can change intracellular and extracellular physiology to cause excitation $[31,32]$. For instance, terminal sustained activation $(5 \mathrm{~min})$ of Arch3.0 was found to cause spontaneous glutamate release $[31,32]$. Moreover, high-intensity laser light raises brain temperature, which can affect neuronal firing [33]. In an in vitro experiment we also observed light-induced depolarization with extended light exposure in Arch3.0 expressing neurons, however we did not observe this effect within 6 sec duration (see Fig. S1). Given this, we believe that the effects we observed are specific to optogenetic inhibition of neuronal function in dmPFC.

One important consideration in interpreting the in vivo electrophysiology data is that there are a relatively low number of behavioral events (i.e. nose-pokes) that were recorded in each individual session. To facilitate comparison between our optogenetic manipulation studies and the recording study, we kept the reinforcement schedule identical between experiments. As such, we used an FR-1 schedule of reinforcement, and kept the nicotine dose the same $(40 \mu \mathrm{g} / \mathrm{kg})$, and the total number of nose-pokes per session was low for a typical electrophysiological experiment, possibly due to tangling of cables and tubing acting as a distraction to the rat. Moreover, we also were left with unequal number of nose-pokes between the extinction and nicotine condition, possibly inflating our results. With this in mind, we took a bootstrapping approach and calculated whether groups had similar variance for the down sampled trials. We conclude that the differences of variance between the nicotine and extinction neuronal populations is not an artefact of different numbers of trials. While the in vivo electrophysiology has some important weaknesses, we believe that our careful analyses addressed all major issues. Therefore, we see these data as important extension of the other findings in this paper, highlighting a critical role of dmPFC neuronal activity in the control of nicotine seeking during cue presentation.

Role of dmPFC in the control of cue-induced drug seeking. The PFC is believed to be involved in maintaining a neural representation of a goal, determining the relative value of each goal and selecting the best course of action to achieve that goal [34]. Reversible inactivation (e.g. muscimol infusions) of mPFC has resulted in perseverative behavior, as well as deficits in task-switching and behavioral flexibility $[35,36]$. Furthermore, different neuronal subpopulations encode different aspects of goal-directed behavior, with the pyramidal neurons displaying the largest amount of heterogeneity [37]. Thus, activity in mPFC is required to maintain top-down control in the face of distracting factors in order to achieve a goal. However, a rapidly changing environment also requires the animal to flexibly change its behavior when necessary [38]. The role of mPFC in this setting is to maintain selective retrieval of memories associated with the current context, while at the same time inhibiting retrieval of unrelated memories [39, 40]. Thus, in our experiments, inhibition of dmPFC neurons during the nicotine cue caused a failure to cease nicotine seeking during extinction, which is consistent with this proposed role for mPFC. However, in our experiments the failure to extinguish ongoing behavior was selective to a nicotine cue, we observed no effect of this manipulation on a food-associated cue.

This would suggest that extinction of food and drug seeking are mediated by distinct neurobiological substrates. Indeed, selfadministration of cocaine and food rewards have been shown to be associated with distinct firing patterns in the nucleus accumbens [41]. Moreover, multiple pharmacological manipulations have been shown to decrease nicotine self-administration, but do not affect responding for a natural reward [42, 43]. In addition, the role of the nicotine-associated cue in nicotine selfadministration is well documented, while food pellet selfadministration can be maintained at high levels without a conditioned stimulus. Given the well-established cue-responsive elements of dmPFC activity, this may explain why this manipulation is specific to nicotine seeking.

Another explanation for the observed behavioral effect may lie in the reinforcing properties of nicotine. Nicotine can act as a primary reinforce and repeated association with nicotine can cause a stimulus to become a conditioned reinforcer, but nicotine is also thought to enhance the incentive value of stimuli which co-occur with nicotine, independent of conditioning [44]. Rather than conditioning, the cue gains incentive value itself. Disruption of the system that processes the cue at the moment of cue presentation, may reduce the incentive value of that cue. Thus, optical inhibition of the dmPFC neurons may have reduced the incentive value of the nicotine cue and the observed increase in responding may result from an effort to compensate for this loss. However, the incentive value of the cue has only been assessed indirectly through levels of responding. To test this hypothesis, studies combining optical interventions targeting the mPFC with direct measures of incentive value such as sign 
tracking in response to a nicotine cue and calculating a response bias score would be necessary [45].

We tested nicotine seeking without optogenetic inhibition the day after the optogenetic inhibition test (Figs. 1d and 3d). This test was designed to determine whether extinction learning is compromised by optogenetic inhibition of dmPFC or vmPFC during the nicotine cue. We found that nicotine seeking on this test data did not differ between the eYFP and Arch3.0 groups. These results indicate that inhibition of either dmPFC or vmPFC neurons during cue presentation does not affect extinction learning, despite the observed increase in nicotine seeking caused by optogenetic inhibition of dmPFC. One prediction from this conclusion is that extinction learning should be impaired by our manipulation, however, we did not observe this. Specifically, nicotine seeking on the (non-stimulated) extinction test session after the first test session was not different between the Arch3.0 and eYFP groups. What might be the cause of this? We believe it is possible that updating the incentive value of the nicotine-associated cue was disrupted, but a different aspect of operant conditioning did undergo between-session extinction. An example would be the actionoutcome contingency, which may be encoded in a brain region other than the dmPFC.

Gutman and colleagues recently used a comparable approach in cocaine trained rats, and they found that response-contingent inhibition of the infralimbic mPFC during extinction sessions increased responding during extinction, as well as during cueinduced reinstatement. In contrast, they show that responsecontingent inhibition of the prelimbic mPFC decreased cocaine seeking during extinction [46]. The discrepancy between this study and ours are most likely based on the inherent differences in maintenance of self-administration of cocaine versus nicotine. In nicotine self-administration, the discrete cue is a critical factor that helps maintain self-administration [44], while cocaine selfadministration can be maintained without a discrete cue [47]. Furthermore, Gutman used no discrete cue during the responsecontingent delivery of $20 \mathrm{~s}$ optogenetic inhibition, whereas we delivered the discrete cue during the response-contingent optical intervention. These procedural differences have the potential to rely on distinct learning mechanisms, and thus potentially change the underlying neurobiological substrates. Future studies are warranted to disentangle the cause underlying the differences between the Gutman study and ours.

We found no evidence that stimulation of dmPFC neurons during cue presentations had an effect on nicotine seeking. It is important to consider the possible alternative explanations for why this manipulation did not decrease nicotine seeking. ChR2mediated stimulation of dmPFC neurons will increase excitation in all ChR2-expressing neurons. Such light-mediated excitation might not effectively mimic the endogenous activity that occurs during the behavioral tests. In contrast, optical inhibition with Arch3.0 is more likely to suppress the activity of neurons that would otherwise be active, with minimal (behaviorally relevant) effects on neurons that are already hyperpolarized. Removal of an input from the dmPFC to one of its projection regions through Arch3.0-mediated inhibition might be sufficient to remove a critical component during the $6-5$ inhibition period. It remains a possibility that different excitation parameters may cause changes in nicotine seeking. However, with the current procedure we are unable to show that excitation of dmPFC neurons during the nicotine cue presentation is sufficient to decrease nicotine seeking.

While optogenetic inhibition of dmPFC during nicotine cue presentation increased nicotine seeking on the first day of extinction, we observed no effect of optogenetic excitation or inhibition of dmPFC during cue exposure on nicotine selfadministration. The fact that responding in the presence of nicotine is unaffected by dmPFC manipulation indicates that nicotine self-administration is not critically dependent on the dmPFC. Mice lacking the $\beta 2$ nicotinic receptor subunit do not self-administer nicotine, while re-expression of the $\beta 2$ subunit in the ventral tegmental area (VTA) is sufficient to rescue nicotine self-administration behavior [48]. Therefore, nicotinic receptors in the $\mathrm{mPFC}$ appear to be not necessary for nicotine reinforcement. However, a recent study showed that activity of the $a 5$ subunit of the nicotinic acetylcholine receptor (nAChR) in dmPFC is associated with hypofrontality [49]. Thus, while there is a critical role for the actions of nicotine in dmPFC in addiction, here we show that cue-induced nicotine seeking is critically dependent on activity of dmPFC during the presentation of the cue.

Role of VmPFC in the control of cue-induced drug seeking. In contrast to the dmPFC, here we found no effect on nicotine seeking from optogenetic inhibition of VmPFC during the nicotine cue. This finding suggests that there is no role of vmPFC in encoding nicotine cues. However, previously we showed that reversible inactivation, via muscimol infusion, of both dmPFC and vmPFC decreases cue-induced reinstatement of nicotine seeking [9]. Therefore a role of vmPFC in mediating cueinduced nicotine seeking has previously been established. We propose that the more specific manipulation we have used here, i.e. optogenetic inhibition of CaMKIla neurons in MPFC during nicotine cue presentation, has revealed a more specific role for dmPFC in representation of the nicotine cue, compared to vmPFC. Thus, we do not rule out a function of vmPFC in nicotine seeking, rather our data support a primary role for dmPFC, and not $\mathrm{VmPFC}$, in representing the incentive value of the nicotineassociated cue.

There is evidence that VmPFC is an important brain region for the inhibitory control over drug seeking, such as that learned through extinction [11, 14]. However, the literature is not conclusive on this matter. While there is good evidence that this is the case for extinction of cocaine seeking [11, 14], comparable studies in rats trained for heroin self-administration have showed that vmPFC activity is critical for promoting relapse [13]. The opposing roles of dmPFC and vmPFC have been the subject of many reviews [14, 15, 50, 51]. Ensemble targeting approaches [52], have shed some light on this debate. For example, Warren et al., [12] used the Daun02 inactivation procedure to show that there are distinct neuronal populations (ensembles) that encode either reward seeking or extinction of reward seeking within the vmPFC. Furthermore, Suto et al. [53] used the Daun02 inactivation procedure to inactivate distinct ensembles within the infralimbic mPFC, causing both suppression or promotion of reward seeking behavior depending on the ensemble that was inactivated. Given that we did not use activity dependent methods to confine expression of Arch3.0, the negative data reported here should be interpreted with caution until comparable experiments are conducted with nicotine seeking rats.

\section{CONCLUSIONS}

Our studies indicate that there is a population of putative glutamatergic neurons within the dorsal mPFC that is activated by nicotine cue exposure and that regulates the incentive value of the cue. This behavioral effect does not seem to simply depend on reduced global activity of the dmPFC, but rather on inhibition coinciding with cue presentation. This neural mechanism does not translate to food-associated cues and to glutamatergic neurons of the vmPFC. Future studies probing the physiological activities and molecular characteristics of cueresponsive neurons in dmPFC during different phases of nicotine taking and seeking will provide further insight into how nicotine cues are processed in the dmPFC. 


\section{FUNDING AND DISCLOSURE}

RFS was funded by a ZonMW Top grant (91209005). The authors declare no competing interests.

\section{ACKNOWLEDGEMENTS}

We thank Drs Michel van den Oever, Antonio Luchicchi and Ouissame Mnie Filali for valuable experimental and technical insight. We would like to express our gratitude to Dr Karl Deisseroth for providing the viral constructs needed to perform the experiments described in this article.

\section{ADDITIONAL INFORMATION}

Supplementary Information accompanies this paper at (https://doi.org/10.1038/ s41386-019-0449-x).

Publisher's note: Springer Nature remains neutral with regard to jurisdictional claims in published maps and institutional affiliations.

\section{REFERENCES}

1. World Health Organization. WHO report on the global tobacco epidemic: raising taxes on tobacco. World Health Organization. 2015. p. 52-53.

2. Franklin TR, Wang Z, Wang J, Sciortino N, Harper D, Li Y, et al. Limbic activation to cigarette smoking cues independent of nicotine withdrawal: a perfusion fMRI study. Neuropsychopharmacology 2007;32:2301-9.

3. Bossert JM, Marchant NJ, Calu DJ, Shaham Y. The reinstatement model of drug relapse: recent neurobiological findings, emerging research topics, and translational research. Psychopharmacol (Berl) 2013;229:453-76.

4. Robinson TE, Yager LM, Cogan ES, Saunders BT. On the motivational properties of reward cues: individual differences. Neuropharmacology. 2014;76 Pt B:450-9.

5. Shaham Y, Shalev U, Lu L, de Wit H, Stewart J. The reinstatement model of drug relapse: history, methodology and major findings. Psychopharmacol (Berl) 2003;168:3-20.

6. Goudriaan $A E$, de Ruiter MB, van den Brink W, Oosterlaan J, Veltman DJ. Brain activation patterns associated with cue reactivity and craving in abstinent problem gamblers, heavy smokers and healthy controls: an fMRI study. Addict Biol 2010;15:491-503.

7. Pich EM, Pagliusi SR, Tessari M, Talabot-Ayer D, Hooft van Huijsduijnen R, Chiamulera $\mathrm{C}$. Common neural substrates for the addictive properties of nicotine and cocaine. Science 1997;275:83-86.

8. Schroeder BE, Binzak JM, Kelley AE. A common profile of prefrontal cortical activation following exposure to nicotine- or chocolate-associated contextual cues. Neuroscience 2001;105:535-45.

9. Lubbers BR, van Mourik Y, Schetters D, Smit AB, De Vries TJ, Spijker S. Prefrontal gamma-aminobutyric acid type $A$ receptor insertion controls cue-induced relapse to nicotine seeking. Biol Psychiatry 2014;76:750-8.

10. Heidbreder CA, Groenewegen HJ. The medial prefrontal cortex in the rat: evidence for a dorso-ventral distinction based upon functional and anatomical characteristics. Neurosci Biobehav Rev 2003;27:555-79.

11. Peters J, LaLumiere RT, Kalivas PW. Infralimbic prefrontal cortex is responsible for inhibiting cocaine seeking in extinguished rats. J Neurosci 2008;28: 6046-53.

12. Warren BL, Mendoza MP, Cruz FC, Leao RM, Caprioli D, Rubio FJ, et al. Distinct Fos-expressing neuronal ensembles in the ventromedial prefrontal cortex mediate food reward and extinction memories. J Neurosci 2016;36:6691-703.

13. Bossert JM, Stern AL, Theberge FR, Cifani C, Koya E, Hope BT, et al. Ventral medial prefrontal cortex neuronal ensembles mediate context-induced relapse to heroin. Nat Neurosci 2011;14:420-2.

14. Peters J, Kalivas PW, Quirk GJ. Extinction circuits for fear and addiction overlap in prefrontal cortex. Learn Mem 2009;16:279-88.

15. Goode TD, Maren S. Common neurocircuitry mediating drug and fear relapse in preclinical models. Psychopharmacology (Berl). 2018; 236:415-37

16. Gipson CD, Reissner KJ, Kupchik YM, Smith AC, Stankeviciute N, Hensley-Simon $M E$, et al. Reinstatement of nicotine seeking is mediated by glutamatergic plasticity. Proc Natl Acad Sci USA 2013;110:9124-9.

17. Kalivas PW. The glutamate homeostasis hypothesis of addiction. Nat Rev Neurosci 2009;10:561-72

18. Van den Oever MC, Rotaru DC, Heinsbroek JA, Gouwenberg $Y$, Deisseroth $K$, Stuber GD, et al. Ventromedial prefrontal cortex pyramidal cells have a temporal dynamic role in recall and extinction of cocaine-associated memory. J Neurosci 2013;33:18225-33.

19. Van den Oever MC, Spijker S, Smit AB. The synaptic pathology of drug addiction. Adv Exp Med Biol 2012;970:469-91.
20. Gipson CD, Kupchik YM, Kalivas PW. Rapid, transient synaptic plasticity in addiction. Neuropharmacology. 2014;76 Pt B:276-86.

21. Van den Oever MC, Goriounova NA, Li KW, Van der Schors RC, Binnekade R, Schoffelmeer AN, et al. Prefrontal cortex AMPA receptor plasticity is crucial for cue-induced relapse to heroin-seeking. Nat Neurosci 2008;11:1053-8.

22. Chen BT, Yau HJ, Hatch C, Kusumoto-Yoshida I, Cho SL, Hopf FW, et al. Rescuing cocaine-induced prefrontal cortex hypoactivity prevents compulsive cocaine seeking. Nature 2013;496:359-62.

23. McFarland K, Kalivas PW. The circuitry mediating cocaine-induced reinstatement of drug-seeking behavior. J Neurosci 2001;21:8655-63.

24. Nagel G, Szellas T, Huhn W, Kateriya S, Adeishvili N, Berthold P, et al. Channelrhodopsin-2, a directly light-gated cation-selective membrane channel. Proc Natl Acad Sci USA 2003;100:13940-5.

25. Chow BY, Han X, Dobry AS, Qian X, Chuong AS, Li M, et al. High-performance genetically targetable optical neural silencing by light-driven proton pumps. Nature 2010;463:98-102.

26. De Vries TJ, de Vries W, Janssen MC, Schoffelmeer AN. Suppression of conditioned nicotine and sucrose seeking by the cannabinoid-1 receptor antagonist SR141716A. Behav Brain Res 2005;161:164-8.

27. Sparta DR, Stamatakis AM, Phillips JL, Hovelso N, van Zessen R, Stuber GD. Construction of implantable optical fibers for long-term optogenetic manipulation of neural circuits. Nat Protoc 2011;7:12-23.

28. Leventhal DK, Gage GJ, Schmidt R, Pettibone JR, Case AC, Berke JD. Basal ganglia beta oscillations accompany cue utilization. Neuron 2012;73:523-36.

29. Bloem B, Schoppink L, Rotaru DC, Faiz A, Hendriks P, Mansvelder HD, et al. Topographic mapping between basal forebrain cholinergic neurons and the medial prefrontal cortex in mice. J Neurosci 2014;34:16234-46.

30. Bartho $\mathrm{P}$, Hirase $\mathrm{H}$, Monconduit L, Zugaro M, Harris KD, Buzsaki G. Characterization of neocortical principal cells and interneurons by network interactions and extracellular features. J Neurophysiol 2004;92:600-8.

31. Raimondo JV, Kay L, Ellender TJ, Akerman CJ. Optogenetic silencing strategies differ in their effects on inhibitory synaptic transmission. Nat Neurosci 2012; 15:1102-4.

32. Wiegert JS, Oertner TG. How (not) to silence long-range projections with light. Nat Neurosci 2016;19:527-8.

33. Stujenske JM, Spellman T, Gordon JA. Modeling the spatiotemporal dynamics of light and heat propagation for in vivo optogenetics. Cell Rep 2015;12:525-34.

34. Wallis JD, Rich EL. Challenges of interpreting frontal neurons during value-based decision-making. Front Neurosci 2011;5:124.

35. Baker PM, Ragozzino ME. Contralateral disconnection of the rat prelimbic cortex and dorsomedial striatum impairs cue-guided behavioral switching. Learn Mem 2014;21:368-79.

36. Ragozzino ME. The contribution of the medial prefrontal cortex, orbitofrontal cortex, and dorsomedial striatum to behavioral flexibility. Ann N Y Acad Sci 2007;1121:355-75.

37. Pinto L, Dan Y. Cell-type-specific activity in prefrontal cortex during goal-directed behavior. Neuron 2015;87:437-50.

38. Miller EK, Cohen JD. An integrative theory of prefrontal cortex function. Annu Rev Neurosci 2001;24:167-202

39. Depue BE. A neuroanatomical model of prefrontal inhibitory modulation of memory retrieval. Neurosci Biobehav Rev 2012;36:1382-99.

40. Xu W, Sudhof TC. A neural circuit for memory specificity and generalization. Science 2013;339:1290-5.

41. Carelli RM, ljames SG, Crumling AJ. Evidence that separate neural circuits in the nucleus accumbens encode cocaine versus "natural" (water and food) reward. J Neurosci. 2000;20:4255-66.

42. Paterson NE, Froestl W, Markou A. Repeated administration of the GABAB receptor agonist CGP44532 decreased nicotine self-administration, and acute administration decreased cue-induced reinstatement of nicotine-seeking in rats. Neuropsychopharmacology 2005;30:119-28.

43. Ramirez-Nino AM, D'Souza MS, Markou A. N-acetylcysteine decreased nicotine self-administration and cue-induced reinstatement of nicotine seeking in rats: comparison with the effects of $\mathrm{N}$-acetylcysteine on food responding and food seeking. Psychopharmacol (Berl). 2013;225:473-82.

44. Caggiula AR, Bevins RA. The Motivational Impact of Nicotine and its Role in Tobacco Use: Chapter 6: The Role of Nicotine in Smoking: A Dual-Reinforcement Model. New York: Springer-Verlag; Vol 55, 2009. p. 91-109.

45. Spoelder M, Tsutsui KT, Lesscher HM, Vanderschuren LJ, Clark JJ. Adolescent alcohol exposure amplifies the incentive value of reward-predictive cues through potentiation of phasic dopamine signaling. Neuropsychopharmacology 2015:40:2873-85.

46. Gutman AL, Nett KE, Cosme CV, Worth WR, Gupta SC, Wemmie JA, et al. Extinction of cocaine seeking requires a window of infralimbic pyramidal neuron activity after unreinforced lever presses. J Neurosci 2017;37: 6075-86. 
47. Broos N, Loonstra R, van Mourik Y, Schetters D, Schoffelmeer AN, Pattij T, et al. Subchronic administration of atomoxetine causes an enduring reduction in context-induced relapse to cocaine seeking without affecting impulsive decision making. Addict Biol 2015;20:714-23.

48. Maskos U, Molles BE, Pons S, Besson M, Guiard BP, Guilloux JP, et al. Nicotine reinforcement and cognition restored by targeted expression of nicotinic receptors. Nature 2005;436:103-7.

49. Koukouli F, Rooy M, Tziotis D, Sailor KA, O'Neill HC, Levenga J, et al. Nicotine reverses hypofrontality in animal models of addiction and schizophrenia. Nat Med 2017;23:347-54

50. Badiani A, Belin D, Epstein D, Calu D, Shaham Y. Opiate versus psychostimulant addiction: the differences do matter. Nat Rev Neurosci 2011;12:685-700.

51. Peters GJ, David CN, Marcus MD, Smith DM. The medial prefrontal cortex is critical for memory retrieval and resolving interference. Learn Mem 2013;20:201-9.

52. Cruz FC, Koya E, Guez-Barber DH, Bossert JM, Lupica CR, Shaham Y, et al. New technologies for examining the role of neuronal ensembles in drug addiction and fear. Nat Rev Neurosci 2013;14:743-54.

53. Suto N, Laque A, De Ness GL, Wagner GE, Watry D, Kerr T, et al. Distinct memory engrams in the infralimbic cortex of rats control opposing environmental actions on a learned behavior. Elife. 2016;5: e21920. 